\title{
Use of Sugammadex in a Patient with Kennedy's Disease Underwent Laparoscopic Gastrectomy
}

\author{
Min Jung Kim ${ }^{1}$, Sun-Joon Bai ${ }^{2}$, Joung Goo Cho ${ }^{1}$, Byunghag Kang ${ }^{2}$ and Na Young Kim*2 \\ ${ }^{1}$ Department of Anesthesiology and Pain Medicine, National Health Insurance Service Ilsan Hospital, Republic of Korea \\ ${ }^{2}$ Department of Anesthesiology and Pain Medicine, Severance Hospital, Anesthesia and Pain Research Institute, Yonsei University College \\ of Medicine, Republic of Korea \\ *Corresponding author: Na Young Kim, Department of Anesthesiology and Pain Medicine, Severance Hospital, Anesthesia and Pain \\ Research Institute, Yonsei University College of Medicine, 50-1 Yonsei-ro, Seodaemun-gu, Seoul 03722, Republic of Korea
}

\begin{abstract}
ARTICLE INFO
Received: 嵊 January 23, 2020

Published: 彗 January 30, 2020

Citation: Min Jung Kim, Sun-Joon Bai, Joung Goo Cho, Byunghag Kang, Na Young Kim. Use of Sugammadex in a Patient with Kennedy's Disease Underwent Laparoscopic Gastrectomy . Biomed J Sci \& Tech Res 25(1)-2020. BJSTR. MS.ID.004157.
\end{abstract}

Abbreviations: KD: Kennedy's Disease; TOF: Train of Four; ICU: Intensive Care Unit

\section{ABSTRACT}

Background: Kennedy's disease (KD) is a rare neuromuscular disorder of the lower motor neurons, characterized by progressive muscle weakness and bulbar muscle atrophy. KD has important clinical features including laryngospasm and high risk of aspiration, as such, general anesthesia is difficult in these patients.

Case: A 52-year-old male with KD was scheduled to undergo laparoscopic radical gastrectomy under general anesthesia. A T1 response was detected $100 \mathrm{~min}$ after initial administration of rocuronium, and a T4 response was detected after a further $30 \mathrm{~min}$. However, the amplitude of the T4 response was low. At the end of surgery, 5 minutes after administration of $2 \mathrm{mg} / \mathrm{kg}$ of sugammadex, the TOF ratio was not achieved; an additional $2 \mathrm{mg} / \mathrm{kg}$ of sugammadex was administered. Nine minutes after initial administration of sugammadex, a T4/T1 ratio of $90 \%$ was achieved.

Conclusion: Complete reversal of neuromuscular blockade was achieved using sugammadex without any neurological and respiratory complications.

Keywords: Sugammadex; Bulbo-Spinal Atrophy, X-Linked; Minimally Invasive Surgical Procedures; Anesthesia; General

\section{Introduction}

Kennedy's disease (KD), otherwise known as spinal and bulbar muscular atrophy, is a rare, $\mathrm{X}$-linked recessive lower motor neuron disease [1]. It is caused by gradually progressive degeneration of the lower motor neurons [1,2]. KD has important clinical features including recurrent and self-limited laryngospasm caused by unknown pathogenesis and high risk for aspiration due to bulbar muscular atrophy and, as such, general anesthesia is difficult in these patients. A recent report described the use of sugammadex for complete reversal of neuromuscular block in a patient with KD who underwent frontal sinusectomy under general anesthesia [3]. In the present report, we described a laparoscopic case, involving complete reversal of neuromuscular blockade using sugammadex and its safety, in a patient with KD undergoing laparoscopic radical gastrectomy under carbon dioxide (CO2) pneumoperitoneum.

\section{Case Report}

A 52-year-old male (weight: $65 \mathrm{~kg}$, height: $165 \mathrm{~cm}$ ) diagnosed with gastric cancer was scheduled to undergo laparoscopic radical gastrectomy under general anesthesia, and a preoperative evaluation was performed. He was diagnosed with hypertension and dyslipidemia, treated for the past 2 years. Ten years previously, he had noticed progressive muscle weakness in the lower limbs and premature exhaustion. One year ago, he started experiencing difficulty with walking, and three months later he was diagnosed with Kennedy's disease (KD). Electromyogram and nerve conduction studies revealed widespread denervation over the cervical, thoracic, and lumbar segments. Genetic testing, the goldstandard diagnostic method for $\mathrm{KD}$, revealed an expanded 54 
CAG repeat. The CAG expansion number in the androgen receptor (AR) gene (>38 repeats) was considered to be pathological. At preoperative physical examination, the patient exhibited grade II weakness in the lower distal limb, grade IV in the upper distal limb, lower limb muscular atrophy, and diminished deep tendon reflexes in upper (brachioradialis muscle) and lower extremities (knee, ankle). He exhibited slurred speech, dysarthria, perioral fasciculation, and tongue fasciculation. However, to date, he had not experienced difficulties with swallowing or laryngospasm. Other clinical findings included gynecomastia and urological problems including dysuria. Preoperative laboratory investigations revealed a high plasma creatine kinase concentration (263 IU/L [normal range 44 245 IU/L]). Other laboratory findings were within the normal range. Additionally, there was a family history of KD; his brothers were affected.

Glycopyrrolate $(0.1 \mathrm{mg})$ was administered immediately before anesthesia as a premedication. After standard monitoring of mean blood pressure, electrocardiogram, oxygen saturation, and anesthetic depth (RD SedLine Sensor, Masimo, Neuchâtel, Switzerland), general anesthesia was induced using $90 \mathrm{mg}$ of propofol and $0.05-0.1 \mu \mathrm{g} / \mathrm{kg} / \mathrm{min}$ of remifentanil. After confirmation of loss of consciousness, closed neuromuscular monitoring was commenced using accelomyography (TOFWatch SX®, Organon Ltd, Ireland) of the right adductor pollicis muscle. The T4/T1 ratio was $97 \%$ before administration of the neuromuscular blocking agent rocuronium. Rocuronium (50 mg) was administered following calibration and stabilization of the train of four (TOF) watch. Left radial artery catheterization was performed to enable continuous blood pressure monitoring and gas analysis. Two minutes later, when the twitch response to TOF stimulation disappeared, endotracheal intubation was performed using a glidescope. Mechanical ventilation was maintained using $50 \%$ oxygen. Anesthesia was maintained with seveflurane (0.8-1.0 age-adjusted minimal alveolar concentration), and remifentanil was infused continuously at a rate of $0.05-0.1 \mu \mathrm{g} / \mathrm{kg} / \mathrm{min}$. The surgical position was reverse Trendelenburg.

A T1 response was detected 100 min after initial administration of rocuronium, and a $\mathrm{T} 4$ response was detected after a further 30 min. However, the amplitude of the T4 response was low. Additional rocuronium was not administered due to delayed recovery of the neuromuscular blockade; the surgical field was not particularly disturbed by the surgical environment such as abdominal wall tightness. There were no adverse events during surgery. Before the end of surgery, $50 \mu \mathrm{g}$ of fentanyl and $3 \mathrm{mg}$ of ramosetron were administered as a rescue analgesic and antiemetic, respectively. At the end of surgery, T4 was detected; however, fade was evident. Five minutes after administration of $2 \mathrm{mg} / \mathrm{kg}$ of sugammadex, the TOF ratio was not achieved. An additional $2 \mathrm{mg} / \mathrm{kg}$ of sugammadex was administered. Nine minutes after initial administration of sugammadex, a T4/T1 ratio of $90 \%$ was achieved and fade could no longer be detected. Administration of sevoflurane and infusion of remifentanil were terminated; we waited an additional 9 min to extubate after confirming the return of spontaneous breathing. The endotracheal tube was removed $18 \mathrm{~min}$ after the initial administration of sugammadex. The duration of anesthesia was $335 \mathrm{~min}$, and the surgical duration was $290 \mathrm{~min}$. The patient was transferred to the intensive care unit (ICU). He was discharged from ICU 2 days after surgery and discharged home 7 days after surgery. No complications developed.

\section{Discussion}

$\mathrm{KD}$ is a genetic neuromuscular disorder that occurs in adults and is classified as a lower motor neuron disease caused by a tandem CAG repeat expansion in the AR gene, located on the $\mathrm{X}$ chromosome $[2,4]$. The degenerative mechanism in the neuron is that the CAG repeat is expressed as a pathogenic protein that accumulates and subsequently causes neuronal degeneration [2]. The average age at onset of KD is in the mid-40s. Early symptoms include muscle cramps, tremor, and twitching [5,6]. Subsequently, lower and upper limb weakness progresses. Bulbar-related symptoms, including dysarthria, nasal speech, and dysphagia due to tongue and facial muscle fasciculation, can present and may progress to aspiration pneumonia [7]. In patients with $\mathrm{KD}$, the respiratory muscles can be involved [2] and, although self-limiting, high rates of laryngospasm (up to $47 \%$ ) occur during daily activities [8]. Life expectancy is reduced due to bulbar and respiratory muscle weakness, although many patients have a normal life span [9].There are potential risk factors with general anesthesia for KD patients, due to muscle weakness, increased sensitivity to non-depolarizing muscle relaxants, and postoperative respiratory failure. Nevertheless, there have been several successful cases of epidural anesthesia in patients with lower motor neuron disease [10]. One case series addressed anesthetic management in six patients using a search of medical records from the Mayo Clinic [11]. A more recent report described the use of sugammadex for complete reversal of neuromuscular block in a patient with KD who underwent frontal sinusectomy under general anesthesia [3]. However, to the best of our knowledge, no reports have addressed the anesthetic management of KD patients undergoing laparoscopic surgery under carbon dioxide pneumoperitoneum.

Sugammadex is the first selective binding agent for the reversal of amino steroid non-depolarizing neuromuscular-blocking agents. It encapsulates with a 1:1 ratio and adequately reverses neuromuscular blocking agents such as rocuronium or vecuronium [12]. Sugammadex provides a rapid and dose-dependent reversal of deep neuromuscular blockade. Several reports have described the safety of sugammadex in patients with neuromuscular disease such as myasthenia gravis [13], amyotrophic lateral sclerosis [14], and Duchenne muscular dystrophy [15]. In the present case, continuous closed neuromuscular monitoring was performed to ensure the degree of the recovery of neuromuscular blockade during long duration of anesthesia ( $5 \mathrm{~h} 3 \mathrm{~min}$ ). At the conclusion of surgery, 260 $\mathrm{mg}$ of sugammadex ( $4 \mathrm{mg} / \mathrm{kg}$ ) was administered for the reversal of 
neuromuscular blockade, after confirmation of a TOF ratio $>0.9$ Extubation was performed successfully without any complications, such as laryngospasm, hypoxia, muscular weakness, or respiratory difficulty. In summary, we successfully managed a patient with KD undergoing a laparoscopic surgical procedure using sugammadex, without any complications. Although deep neuromuscular blockade could not be maintained throughout the surgery, we propose that sugammadex may be used to safely manage patients with lower motor disease, such as KD, in long-duration, laparoscopic surgical procedures involving general anesthesia.

\section{Funding}

None

\section{Disclosures/Conflict of Interest}

The authors declare that there is no conflict of interest.

\section{References}

1. Grunseich C, Fischbeck KH (2015) Spinal and Bulbar Muscular Atrophy Neurol Clin 33: 847-854

2. Querin G, Soraru G, Pradat PF (2017) Kennedy disease (X-linked recessive bulbospinal neuronopathy): A comprehensive review from pathophysiology to therapy. Rev Neurol (Paris) 173: 326-337.

3. Takeuchi R, Hoshijima H, Doi K, Nagasaka H (2014) The use of sugammadex in a patient with Kennedy's disease under general anesthesia. Saudi J Anaesth 8: 418-420.

4. Choi WT, Mac Lean HE, Chu S, Warne GL, Zajac JD (1993) Kennedy's disease: genetic diagnosis of an inherited form of motor neuron disease. Aust N Z J Med 23: 187-192.

\section{ISSN: 2574-1241}

DOI: 10.26717/BJSTR.2020.25.004157

Na Young Kim. Biomed J Sci \& Tech Res

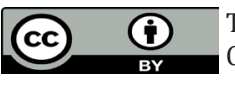

This work is licensed under Creative ommons Attribution 4.0 License

Submission Link: https://biomedres.us/submit-manuscript.php
5. Sperfeld AD, Karitzky J, Brummer D, Schreiber H, Haussler J, et al. (2002) X-linked bulbospinal neuronopathy: Kennedy disease. Arch Neurol 59: 1921-1926.

6. Lee JH, Shin JH, Park KP, Kim IJ, Kim CM, et al. (2005) Phenotypic variability in Kennedy's disease: implication of the early diagnostic features. Acta Neurol Scand 112: 57-63.

7. Warnecke T, Oelenberg S, Teismann I, Suntrup S, Hamacher C, et al. (2009) Dysphagia in X-linked bulbospinal muscular atrophy (Kennedy disease). Neuromuscul Disord 19: 704-708.

8. Sperfeld AD, Hanemann CO, Ludolph AC, Kassubek J (2005) Laryngospasm: an underdiagnosed symptom of X-linked spinobulbar muscular atrophy. Neurology 64: 753-754.

9. Finsterer J (2010) Perspectives of Kennedy's disease. J Neurol Sci 298: 1-10.

10. Okamoto E, Nitahara K, Yasumoto M, Higa K (2004) Use of epidural anaesthesia for surgery in a patient with Kennedy's disease. Br J Anaesth 92: 432-433.

11. Niesen AD, Sprung J, Prakash YS, Watson JC, Weingarten TN (2009) Case series: anesthetic management of patients with spinal and bulbar muscular atrophy (Knedy's disease). Can J Anaesth 56: 136-141.

12. Yang LP, Keam SJ (2009) Sugammadex: A review of its use in anaesthetic practice. Drugs 69: 919-942.

13. Unterbuchner C, Fink H, Blobner M (2010) The use of sugammadex in a patient with myasthenia gravis. Anaesthesia 65: 302-305.

14. Wachi M, Uehara K, Fujinaka W, Takatori M, Tada K (2011) [Use of sugammadex in a patient with amyotrophic lateral sclerosis]. Masui 60: 1408-1410.

15. De Boer HD, Van Esmond J, Booij LH, Driessen JJ (2009) Reversal of rocuronium-induced profound neuromuscular block by sugammadex in Duchenne muscular dystrophy. Paediatr Anaesth 19: 1226-1228.

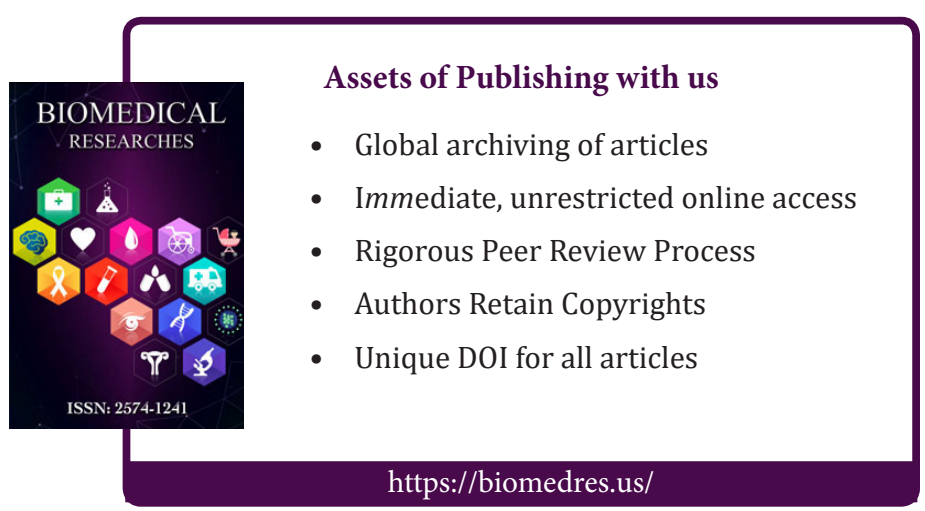

